\title{
Association Between the Presence or Severity of Coronary Artery Disease and Pericardial Fat, Paracardial Fat, Epicardial Fat, Visceral Fat, and Subcutaneous Fat as Assessed by Multi-Detector Row Computed Tomography
}

\author{
Yoko Ueda, ${ }^{1}$ MD, Yuhei Shiga, ${ }^{1}$ MD, Yoshiaki Idemoto, ${ }^{1}$ MD, Kohei Tashiro, ${ }^{1}$ MD, Kota Motozato, ${ }^{1}$ MD,
} Rie Koyoshi, ${ }^{1}$ MD, Takashi Kuwano, ${ }^{1}$ MD, Kanta Fujimi, ${ }^{1,2}$ MD, Masahiro Ogawa, ${ }^{1}$ MD, Keijiro Saku, ${ }^{1,3}$ MD and Shin-ichiro Miura, ${ }^{1,3}$ MD

\begin{abstract}
Summary
The associations between the presence or severity of coronary artery disease (CAD) and measurements of various kinds of fat as assessed by multidetector row computed tomography (MDCT) are unclear. We enrolled 300 patients who were clinically suspected to have CAD or who had at least one cardiac risk factor and had undergone MDCT. The number of significantly stenosed coronary vessels (VD), and measurements of pericardial fat index, paracardial fat index, epicardial fat index, visceral fat index, and subcutaneous fat index were quantified using MDCT. Plasma levels of adiponectin, pentaxin-3, and high-sensitivity C-reactive protein factors were also measured. Pericardial fat index, paracardial fat index, and visceral fat index in a CAD group were significantly greater than those in a non-CAD group. In addition, the levels of these fat indices tended to increase as the number of VD increased and were positively correlated with the Gensini score. The area-underthe-curve for paracardial fat index was significantly greater than those for the other parameters of fat index measured by a receiver-operating characteristic curve analysis. The cut-off level of paracardial fat index that gave the greatest sensitivity and specificity for the diagnosis of CAD was $54.9 \mathrm{~cm}^{3} / \mathrm{m}^{2}$ (sensitivity 0.710 , specificity 0.552). The presence of CAD was independently associated with paracardial fat index, in addition to age and diabetes mellitus, by a multiple logistic regression analysis. In conclusion, paracardial fat index may be a marker for evaluating the presence or severity of CAD.
\end{abstract}

(Int Heart J 2018; 59: 695-704)

Key words: Pericardial fat index, Paracardial fat index, Epicardial fat index, Visceral fat index, Subcutaneous fat index

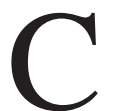
oronary artery disease (CAD) is mainly caused by arteriosclerosis. There are various risk factors for CAD, such as hypertension (HTN), diabetes mellitus (DM), dyslipidemia (DL), and metabolic syndrome (MetS). In Japan, MetS is diagnosed according to the modified guidelines as visceral fat area $\geq 100 \mathrm{~cm}^{2}$ and the presence of two or more of the following: high blood pressure (BP) [systolic BP $(\mathrm{SBP}) \geq 130 \mathrm{mmHg}$ or diastolic BP (DBP) $\geq 85 \mathrm{mmHg}$ or taking an antihypertensive drug], DL [triglycerides $(\mathrm{TG}) \geq 150 \mathrm{mg} / \mathrm{dL}$ or $40 \mathrm{mg} / \mathrm{dL}>$ high-density lipoprotein cholesterol (HDLC) or high fasting glucose (fasting glucose $\geq 110 \mathrm{mg} / \mathrm{dL}$ or taking a glucose-lowering drug). ${ }^{1)}$ Many studies have shown that visceral fat has a detrimental effect on metabolism and the risk of CAD. ${ }^{2-4)}$ The best tool for estimating visceral fat is multi-detector row computed tomography (MDCT). MDCT has become more widely available in many general hospitals, and enables the accurate noninvasive assessment of coronary artery stenosis, ${ }^{5)}$ calcification, ${ }^{6)}$ and plaque imaging. ${ }^{7)}$

\section{Editorial p.671}

Recently, ectopic fat has attracted attention. Surplus ectopic fat accumulates in several organs, such as the heart, liver and muscle, which contain just a small amount of adipose tissue. The fat located around the heart is called pericardial fat. Pericardial fat consists of paracardial fat and epicardial fat. Paracardial fat is located on the epicardium surface and epicardial fat is located between the epicardium and myocardium, and each type of cardial fat can be scanned by MDCT. Several studies have supported the notion that ectopic fat is associated with MetS and insulin resistance, ${ }^{8)}$ and a positive correlation has been

From the ${ }^{1}$ Department of Cardiology, Fukuoka University School of Medicine, Fukuoka, Japan, ${ }^{2}$ Department of Rehabilitation, Fukuoka University Hospital, Fukuoka, Japan and ${ }^{3}$ Department of Molecular Cardiovascular Therapeutics, Fukuoka University School of Medicine, Fukuoka, Japan.

Address for correspondence: Shin-ichiro Miura, MD, Department of Cardiology, Fukuoka University School of Medicine, 7-45-1 Nanakuma, Jonan-ku, Fukuoka, 814-0180, Japan. E-mail: miuras@ cis.fukuoka-u.ac.jp

Received for publication April 21, 2017. Revised and accepted September 11, 2017.

Released in advance online on J-STAGE June 6, 2018.

doi: 10.1536/ihj.17-234

All rights reserved by the International Heart Journal Association. 
shown to exist between pericardial fat and visceral fat. ${ }^{9)}$ However, the association between the presence or severity of CAD and pericardial fat is unclear, and it is not known which types of fats are most closely associated with the presence or severity of CAD.

Therefore, we determined, among the levels of pericardial fat, paracardial fat, epicardial fat, visceral fat, and subcutaneous fat, which is most closely associated with the presence and severity of CAD.

\section{Methods}

Study Subjects: Three hundred consecutive subjects who were clinically suspected of having CAD or who had at least one cardiac risk factor were enrolled in this study. All subjects underwent MDCT coronary angiography between April 2012 and July 2014. Patients with serum creatinine $>2.0 \mathrm{mg} / \mathrm{dL}$ or contrast-induced allergy did not undergo MDCT. The protocol in this study was approved by the ethics committee of Fukuoka University Hospital [IRB \#11-06(09-089)], and all subjects gave their informed consent to participate.

Evaluation of coronary stenosis using MDCT: We evaluated coronary stenosis using MDCT as previously described. ${ }^{10)}$ Of the 300 patients, 208 who underwent MDCT were scanned by 64-MDCT on an Aquilion 64 (Toshiba, Tokyo), and 92 patients who underwent MDCT were scanned by 320-MDCT on an Aquilion ONE ViSION (Toshiba, Tokyo). The use of a beta-blocker and nitroglycerin before scanning was left to the physician's discretion. In the first MDCT, a $70 \mathrm{~mL}$ bolus of contrast medium (Omnipaque, $350 \mathrm{mg}$ iodine/mL; Daiichi Sankyo Co., Ltd., Tokyo) was injected at a flow rate of $3.6 \mathrm{~mL} /$ sec, followed by $35 \mathrm{~mL}$ contrast agent and $30 \mathrm{~mL}$ saline solution, each at a flow rate of $1.8 \mathrm{~mL} / \mathrm{sec}$, with a dual injector. In the second MDCT, $21.5 \mathrm{mgI} / \mathrm{kg} / \mathrm{second}$ contrast medium (Iopamiron, $370 \mathrm{mg}$ iodine/mL; Bayer Yakuhin Ltd, Osaka, Japan) equivalent to the patient's body weight $\times 0.7 \mathrm{~mL}$ was injected over 10 seconds, followed by 35 $\mathrm{mL}$ contrast agent and $30 \mathrm{~mL}$ saline solution, each at a flow rate of $1.8 \mathrm{~mL} / \mathrm{second}$, with a dual injector.

The region of interest was placed within the ascending aorta, and the scan was started when the CT density reached 100 Hounsfield units higher than the baseline CT density. The scan was performed between the tracheal bifurcation and diaphragm with the following parameters: 64-MDCT-collimation width $0.5 \mathrm{~mm}$, rotation speed 0.4 seconds/rotation, tube voltage $135 \mathrm{kV}$, and effective tube current $360 \mathrm{~mA}$; 320-MDCT-collimation width $0.5 \mathrm{~mm}$, rotation speed 0.275 seconds/rotation, tube voltage 120 $\mathrm{kV}$, and auto tube current.

Overall, 15 coronary artery segments were assessed in all patients. Narrowing of the normal contrast-enhanced lumen to $\geq 50 \%$ that could be identified in multiplanar reconstructions or cross-sectional images was defined as significant stenosis in CAD. In addition, in all patients, the atherosclerotic severity of coronary artery disease was assessed in terms of the Gensini score. ${ }^{11,12)}$

Measurement of pericardial fat, paracardial fat, epicardial fat, visceral fat, and subcutaneous fat: CT scans were performed with a MDCT and a workstation on a Ziostation (Ziosoft Inc., Tokyo). Pericardial fat was defined as pixels within a window level of -195 to -45 , and the volume of fat was measured from the bifurcation of the pulmonary artery to the diaphragm. ${ }^{3,13)}$ The pericardium was traced manually. Intrapericardial fat was defined as epicardial fat, and fat outside the pericardium was defined as paracardial fat. Figure 1 shows representative images obtained using 64-MDCT and 320-MDCT. In addition, to measure the areas of visceral fat and subcutaneous fat, as well as waist circumference (WC), a CT scan was also performed. These values were measured from CT cross-sectional scans at the level of the umbilicus with a workstation on a Ziostation (Ziosoft Inc.). Each fat index was obtained by dividing the value for each type of fat by the body surface area.

Evaluation of risk factors for CAD: Body mass index (BMI), SBP, DBP, serum levels of total cholesterol (TC), TG, HDL-C, low-density lipoprotein cholesterol (LDL-C), ratio of LDL-C to HDL-C (L/H-C), non HDL-C (TC minus HDL-C), uric acid (UA), fasting glucose, hemoglobin A1c (HbA1c), high-sensitivity C-reactive protein (hsCRP), adiponectin, pentraxin 3 (PTX-3), smoking status (current versus nonsmokers), family history [myocardial infarction (MI), angina pectoris or sudden death] and medication use were collected as risk factors in all patients. Plasma levels of adiponectin and PTX-3 were determined in duplicate by specific enzyme immunoassays (R\&D Systems, Minneapolis, MN) according to the manufacturer's protocol.

BMI was calculated as weight $(\mathrm{kg}) /$ height $(\mathrm{m})^{2}$. BP was determined as the mean of two measurements obtained in an office setting by the conventional cuff method using a mercury sphygmomanometer after at least 5 minutes of rest. All of the blood samples were drawn in the morning after the patient had fasted overnight. The patient characteristics were obtained from medical records with regard to history of HTN, DL, DM, and history of smoking. Patients who had a current SBP/DBP $\geq 140 / 90$ $\mathrm{mmHg}$ or who were receiving antihypertensive therapy were considered to have HTN. Patients with LDL-C $\geq 140$ $\mathrm{mg} / \mathrm{dL}, \mathrm{TG} \geq 150 \mathrm{mg} / \mathrm{dL}$, and/or HDL-C $<40 \mathrm{mg} / \mathrm{dL}$ or who were receiving lipid-lowering therapy were considered to have DL. ${ }^{14)} \mathrm{DM}$ was defined using the American Diabetes Association criteria ${ }^{15)}$ or the administration of a glucose-lowering drug. Hyperuricemia (HU) was defined as a serum uric acid level of $\geq 7.0 \mathrm{mg} / \mathrm{dL}$ or the administration of uric acid-lowering drugs.

Statistical analysis: A statistical analysis was performed using Excel 2016 (SSRI, Tokyo), the Stat View statistical software package (Stat View 5; SAS Institute Inc, Cary, NC, USA) and EZR (Saitama Medical Center, Jichi Medical University, Saitama, Japan). Continuous variables are shown as the mean \pm standard deviation. Categorical and continuous variables were compared between the groups by a chi-square analysis and t-test, respectively. A receiver-operating characteristic (ROC) curve analysis was used to determine the cut-off levels of pericardial fat index, paracardial fat index, epicardial fat index, visceral fat index, and subcutaneous fat index to distinguish between the presence and absence of CAD at the highest possible sensitivity and specificity levels. Multivariate analysis was performed by a logistic regression analysis for independ- 

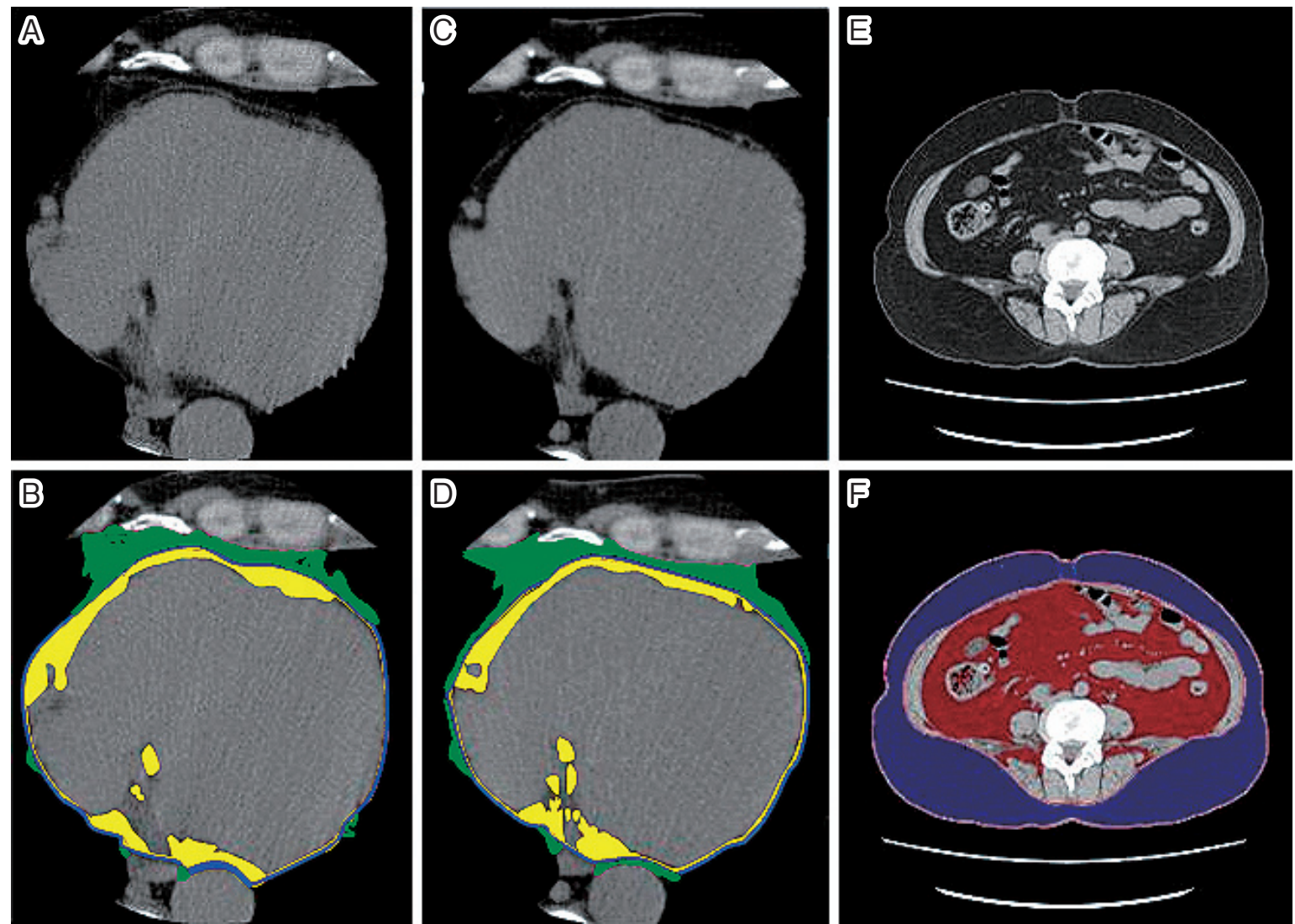

Figure 1. A-B and C-D were obtained by 64-MDCT and 320-MDCT, respectively. The area of fat was defined as pixels within a window level of -195 to -45. In $\mathbf{B}$ and $\mathbf{D}$, blue lines indicate epicardium. Intraepicardial fat shown in yellow is defined as epicardial fat. The region outside the epicardium shown in green is defined as paracardial fat. The total amount of epicardial fat and paracardial fat is defined as pericardial fat. $\mathbf{E}$ and $\mathbf{F}$ were obtained by 320-MDCT. The red and blue areas indicate visceral fat and subcutaneous fat, respectively.

ent variables that were related to the presence or absence of CAD. A value of $P<0.05$ was considered significant.

\section{Results}

Patient characteristics in all patients and the CAD and non-CAD groups: Table I shows the characteristics of the 300 patients $(150(50 \%)$ men and 150 (50\%) women). The frequencies of HTN, DL and DM in all patients were $73 \%, 65 \%$ and $23.7 \%$, respectively. The mean age was 68 \pm 11 years, BMI was $24 \pm 4 \mathrm{~kg} / \mathrm{m}^{2}$, and visceral fat index was $113 \pm 60 \mathrm{~cm}^{2} / \mathrm{m}^{2}$. The volumes of pericardial fat index, paracardial fat index and epicardial fat index were $111 \pm 44 \mathrm{~cm}^{3} / \mathrm{m}^{2}, 54 \pm 23 \mathrm{~cm}^{3} / \mathrm{m}^{2}$, and $58 \pm 28 \mathrm{~cm}^{3} / \mathrm{m}^{2}$, respectively.

There were significant differences in patient characteristics between the CAD and non-CAD groups. The CAD group showed a significantly higher age, \% HT, SBP, \% DM, fasting glucose, HbAlc, \% DL, TG, L/H-C, visceral fat index, \% MetS, hsCRP, pericardial fat index, paracardial fat index, and epicardial fat index, and significantly lower \% females and HDL-C levels than the nonCAD group.

Table II shows the medications in all patients and in the $\mathrm{CAD}$ and non-CAD groups. The percentages of the use of an angiotensin II receptor blocker (ARB)/ angiotensin-converting enzyme inhibitor (ACEI), calcium channel blocker (CCB) and statin in all patients were $42 \%, 37 \%$ and $32 \%$, respectively. There were significant differences in medications between the CAD and nonCAD groups. The CAD group showed significantly higher use of ARB/ACEI, CCB, statin, $\beta$-blocker, sulfonylurea (SU), $\alpha$-glucosidase inhibitor $(\alpha$-GI) and dipeptidyl peptidase-4 inhibitor (DPP-4I) than the non-CAD group.

Measurements of pericardial fat index, paracardial fat index, epicardial fat index, visceral fat index, subcutaneous fat index in the CAD and non-CAD groups: As shown in Figure 2, we analyzed whether there were differences in the pericardial fat index, paracardial fat index, epicardial fat index, visceral fat index and subcutaneous fat index between the CAD and non-CAD groups. The pericardial fat $(P=0.0008)$, paracardial fat $(P<0.0001)$ and visceral fat indices $(P=0.008)$ in the CAD group were significantly higher than those in the non-CAD group.

Association between pericardial fat index, paracardial fat index, epicardial fat index, visceral fat index, or subcutaneous fat index and the number of significantly stenosed coronary vessels (VD): The subjects were divided into 4 groups (0,1, 2 and 3VD groups) according 
Table I. Patient Characteristics in All Patients and in the CAD and Non-CAD Groups

\begin{tabular}{|c|c|c|c|c|}
\hline & $\begin{array}{l}\text { All patients } \\
(n=300)\end{array}$ & $\begin{array}{c}\text { CAD } \\
(n=166)\end{array}$ & $\begin{array}{c}\text { non-CAD } \\
(n=134)\end{array}$ & $\begin{array}{c}\text { CAD versus } \\
\text { non-CAD } \\
P\end{array}$ \\
\hline Age, years & $68 \pm 11$ & $69 \pm 10$ & $63 \pm 13$ & $<0.001$ \\
\hline Gender (male), $\%$ & 50 & 41 & 61 & $<0.001$ \\
\hline Family history, $\%$ & 27 & 29 & 28 & 0.941 \\
\hline Smoking, $\%$ & 44 & 86 & 57 & 0.005 \\
\hline BMI, $\mathrm{kg} / \mathrm{m}^{2}$ & $24 \pm 4$ & $24.0 \pm 3.6$ & $23.9 \pm 3.5$ & 0.800 \\
\hline HTN, \% & 73 & 80 & 63 & $<0.001$ \\
\hline $\mathrm{SBP}, \mathrm{mmHg}$ & $136 \pm 19$ & $138 \pm 20$ & $132 \pm 17$ & 0.004 \\
\hline $\mathrm{DBP}, \mathrm{mmHg}$ & $77 \pm 13$ & $78 \pm 12$ & $76 \pm 13$ & 0.199 \\
\hline DM, \% & 24 & 31 & 14 & $<0.001$ \\
\hline $\mathrm{HbA} 1 \mathrm{c}, \%$ & $6.0 \pm 1.0$ & $6.1 \pm 1.0$ & $5.8 \pm 0.8$ & 0.004 \\
\hline Fasting glucose, $\mathrm{mg} / \mathrm{dL}$ & $109 \pm 29$ & $114 \pm 34$ & $103 \pm 19$ & 0.001 \\
\hline $\mathrm{DL}, \%$ & 65 & 71 & 57 & 0.012 \\
\hline $\mathrm{TG}, \mathrm{mg} / \mathrm{dL}$ & $137 \pm 78$ & $149 \pm 78$ & $125 \pm 76$ & 0.010 \\
\hline HDL-C, mg/dL & $54 \pm 16$ & $52 \pm 15$ & $59 \pm 16$ & $<0.001$ \\
\hline LDL-C, mg/dL & $112 \pm 31$ & $114 \pm 30$ & $112 \pm 32$ & 0.584 \\
\hline L/H-C & $2.2 \pm 0.9$ & $2.4 \pm 0.9$ & $2.0 \pm 0.8$ & 0.026 \\
\hline Non HDL-C & $144.2 \pm 34$ & $145.9 \pm 35$ & $142 \pm 33$ & 0.326 \\
\hline $\mathrm{HU}, \%$ & 15 & 18 & 12 & 0.148 \\
\hline Uric acid, mg/dL & $5.2 \pm 1.33$ & $5.2 \pm 1.2$ & $5.4 \pm 1.3$ & 0.987 \\
\hline MetS, \% & 44 & 54 & 35 & 0.001 \\
\hline $\mathrm{WC}, \mathrm{cm}$ & $86 \pm 10$ & $88 \pm 10$ & $87 \pm 9$ & 0.192 \\
\hline Visceral fat index, $\mathrm{cm}^{2} / \mathrm{m}^{2}$ & $113 \pm 60$ & $125 \pm 63$ & $104 \pm 53$ & 0.003 \\
\hline Subcutaneous fat index, $\mathrm{cm}^{2} / \mathrm{m}^{2}$ & $154 \pm 80$ & $153 \pm 79$ & $162 \pm 82$ & 0.326 \\
\hline Pericardial fat index, $\mathrm{cm}^{3} / \mathrm{m}^{2}$ & $111 \pm 44$ & $119 \pm 42$ & $102 \pm 44$ & 0.001 \\
\hline Paracardial fat index, $\mathrm{cm}^{3} / \mathrm{m}^{2}$ & $54 \pm 23$ & $59 \pm 24$ & $48 \pm 19$ & $<0.001$ \\
\hline Epicardial fat index, $\mathrm{cm}^{3} / \mathrm{m}^{2}$ & $58 \pm 28$ & $60 \pm 25$ & $55 \pm 30$ & 0.072 \\
\hline hsCRP, mg/L & $0.20 \pm 0.46$ & $0.26 \pm 0.57$ & $0.13 \pm 0.24$ & 0.020 \\
\hline Adiponectin, $\mu \mathrm{g} / \mathrm{mL}$ & $7.49 \pm 5.59$ & $7.27 \pm 5.84$ & $7.77 \pm 5.27$ & 0.451 \\
\hline PTX-3, ng/mL & $2.59 \pm 2.27$ & $2.76 \pm 2.77$ & $2.38 \pm 1.40$ & 0.152 \\
\hline
\end{tabular}

Continuous variables are expressed as mean \pm SD. CAD indicates coronary artery disease; BMI, body mass index; HTN, hypertension; SBP, systolic blood pressure; DBP, diastolic blood pressure; DM, diabetes mellitus; DL, dyslipidemia; TG, triglyceride; HDL-C, high density lipoprotein cholesterol; LDL-C, low density lipoprotein cholesterol; L/H-C, a ratio of LDL-C to HDL-C; Non HDL-C, total cholesterol minus HDL-C; HU, hyperuricemia; MetS, metabolic syndrome; WC, waist circumference; VFA, visceral fat area; SFA, subcutaneous fat area; HsCRP, high-sensitivity CRP; and PTX-3, pentraxin-3.

to the number of significantly stenosed coronary vessels (Figure 3). Pericardial fat index, paracardial fat index, epicardial fat index, and visceral fat index, but not subcutaneous fat index, increased slightly as the number of VD increased. Subjects with multi-VD (2 and 3 VD) had significantly higher paracardial fat and visceral fat indices than those with 0 VD (data not shown).

Association between pericardial fat index, paracardial fat index, epicardial fat index, visceral fat index, or subcutaneous fat index and Gensini score: Pericardial fat index $(P=0.0005)$, paracardial fat index $(P<0.0001)$, epicardial fat index $(P=0.026)$, and visceral fat index $(P$ $=0.0008)$, but not subcutaneous fat index $(P=0.197)$, were positively correlated with the Gensini score (Figure 4).

Association between pericardial fat, paracardial fat index or epicardial fat index and visceral fat index or subcutaneous fat index: Pericardial fat, paracardial fat, and epicardial fat indices were positively correlated with visceral fat index (Figure 5A-C). Although pericardial fat index, epicardial fat index, and paracardial fat index were positively correlated with subcutaneous fat index (Figure 5 $\mathrm{D}-\mathrm{F})$, the $\mathrm{r}$ values were very low. Pericardial fat index, paracardial fat index, and epicardial fat index were positively correlated with each other (Figure 5G-I).

Cut-off values of pericardial fat index, paracardial fat index, epicardial fat index, visceral fat index or subcutaneous fat index for the diagnosis of CAD: Since pericardial fat index, paracardial fat index, epicardial fat, and visceral fat index are associated with each other (Figure 5), we performed an ROC curve analysis to determine which type of fat is most closely related to the presence of CAD. This ROC curve analysis showed that the areaunder-the-curve (AUC) of paracardial fat index (AUC 0.650 , sensitivity 0.710 , specificity 0.552 ) was greater than those of pericardial fat index $(0.629,0.611,0.638)$, epicardial fat index $(0.590,0.527,0.663)$, visceral fat index $(0.594,0.756,0.436)$, and subcutaneous fat index $(0.543,0.718,0.387)$ (Figure 6). The paracardial fat index level might be a more useful marker than pericardial fat index, epicardial fat index, visceral fat index, and subcutaneous fat index because paracardial fat index had the 
highest AUC, although there was a small difference in AUC among the 5 types of fat index. The cut-off level of paracardial fat index that gave the greatest sensitivity and specificity for the diagnosis of CAD was $54.9 \mathrm{~cm}^{3} / \mathrm{m}^{2}$.

Predictors of the presence of CAD, including paracardial fat index: We analyzed the predictors of the presence of CAD in all patients using independent variables by logistic regression analysis (Table III). We selected conventional coronary risk factors (gender, age, BMI, HTN, DL, $\mathrm{DM}$ and smoking) and paracardial fat index as variables. The presence of CAD was independently associated with

Table II. Medication in All Patients, the Non-CAD and CAD Groups

\begin{tabular}{lrrrc}
\hline & $\begin{array}{c}\text { All patients } \\
(n=300)\end{array}$ & $\begin{array}{c}\text { CAD } \\
(n=166)\end{array}$ & $\begin{array}{c}\text { non-CAD } \\
(n=134)\end{array}$ & $\begin{array}{c}\text { non-CAD versus } \\
\text { CAD }\end{array}$ \\
\hline ARB/ACE-I, \% & 41.8 & 48.5 & 33.6 & 0.093 \\
CCB, \% & 36.7 & 42.4 & 30.0 & 0.025 \\
Statin, \% & 32.0 & 37.8 & 25.4 & 0.022 \\
$\beta$-blocker, \% & 10.7 & 14.5 & 6.0 & 0.017 \\
DU, \% & 9.4 & 10.9 & 7.5 & 0.311 \\
$\alpha$-blocker, \% & 4.0 & 5.5 & 2.2 & 0.160 \\
Fibrate, \% & 2.0 & 2.4 & 1.5 & 0.564 \\
EPA, \% & 2.0 & 2.4 & 1.5 & 0.569 \\
SU, \% & 7.7 & 11.5 & 3.0 & 0.005 \\
$\alpha-$-GI, \% & 4.3 & 6.7 & 1.5 & 0.029 \\
Biguanide, \% & 7.4 & 9.7 & 4.5 & 0.086 \\
Thiazolidine, \% & 1.3 & 1.2 & 1.5 & 0.834 \\
DPP-4I, \% & 11.0 & 15.2 & 6.0 & 0.012 \\
Insulin, \% & 3.3 & 3.6 & 3.0 & 0.756 \\
\hline
\end{tabular}

Continuous variables are expressed as mean \pm SD. CAD indicates coronary artery disease; ARB, angiotensin II receptor blocker; ACEI, angiotensin-converting enzyme inhibitor; $\mathrm{CCB}$, calcium channel blocker; DU, diuretic; EPA, eicosapentaenoic acid; SU, sulfonylurea; $\alpha$-GI, $\alpha$ glucosidase inhibitor; and DPP-4, dipeptidyl peptidase-4 inhibitor. paracardial fat index $(P=0.015)$, in addition to age $(P<$ $0.001)$, gender $(P=0.019)$, and DM $(P=0.010)$. We also analyzed the predictors of the presence of CAD in all patients using independent variables including conventional coronary risk factors and pericardial fat index (Supplemental Table I), epicardial fat index (Supplemental Table II), or visceral fat index (Supplemental Table III) by logistic regression analysis. Finally, the presence of CAD was not associated with pericardial fat $(P=0.115)$, epicardial fat $(P=0.632)$, or visceral fat $(P=0.669)$.

\section{Discussion}

In the present study, we investigated the association between pericardial fat index, paracardial fat index, epicardial fat index, visceral fat index, or subcutaneous fat index and the presence or severity of CAD as assessed by MDCT. Pericardial fat index, paracardial fat index, epicardial fat index, and visceral fat index, but not subcutaneous fat index, were associated with the presence or severity of CAD. In particular, the presence of CAD was independently associated with paracardial fat index in addition to age, gender and DM by a multiple logistic regression analysis.

Several studies have shown that epicardial fat was associated with CAD risk and coronary atherosclerosis. ${ }^{316-18)}$ Although Rosito, et $a l^{3}{ }^{3)}$ analyzed the association between epicardial fat or paracardial fat and vascular calcification, they did not consider the presence of significant coronary stenosis. Three other studies did not analyze various kinds of fat including paracardial fat and visceral fat. ${ }^{16-18)}$ Rodriguez-Granillo, et al. reported that pericardial fat volume was associated with the atherosclerotic plaque burden rather than the severity of lesions in coronary arteries. ${ }^{19}$ They did not analyze epicardial fat or paracardial fat. In addition, Ghaderi, et al. reported that epicardial fat thick-

\section{A. Pericardial fat index}

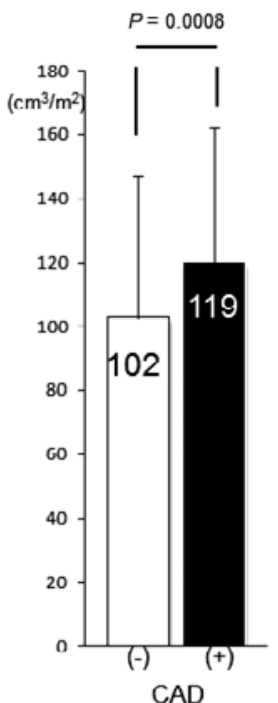

B. Paracardial fat index

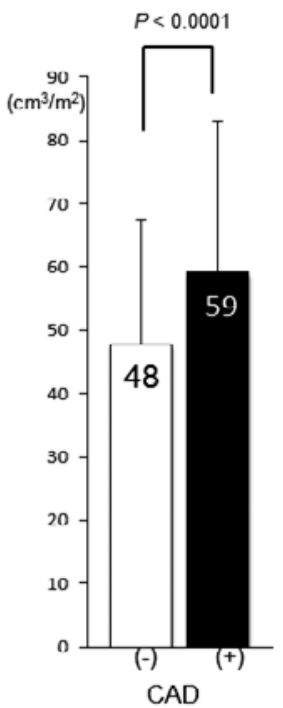

C. Epicardial fat index

D. Visceral fat index

E. Subcutaneous fat index
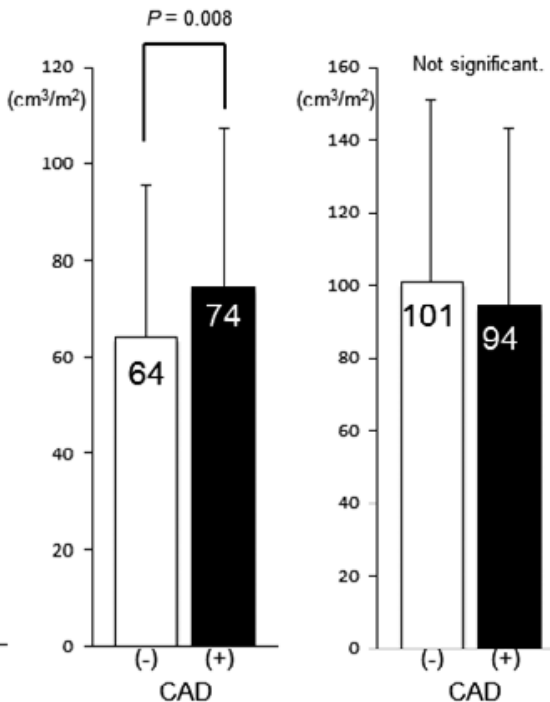

Figure 2. Pericardial fat (A), paracardial fat $(\mathbf{B})$, epicardial fat $(\mathbf{C})$, visceral fat $(\mathbf{D})$ and subcutaneous fat $(\mathbf{E})$ in patients in the nonCAD group (open bars) and in those who had at least one significant CAD (closed bars). NS indicates not significant. 
A. Pericardial fat index

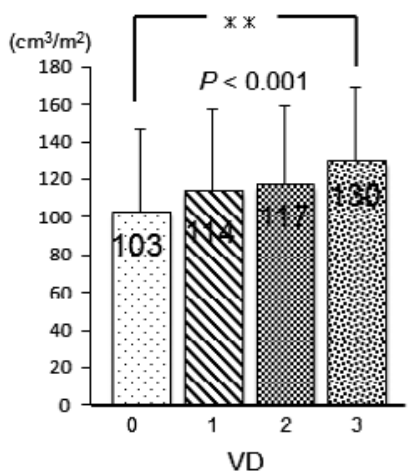

D. Visceral fat index

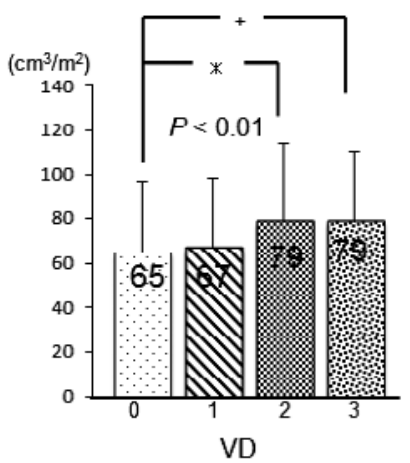

B. Paracardial fat index

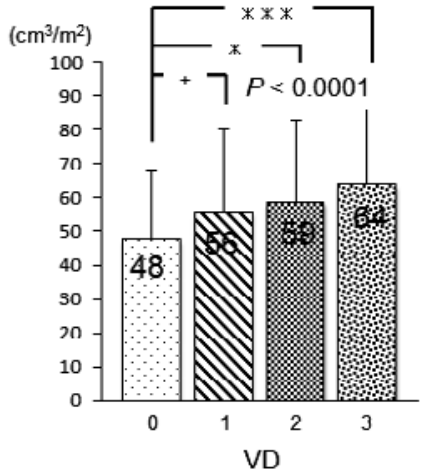

C. Epicardial fat index

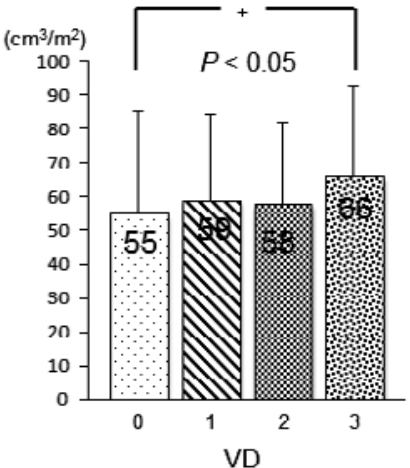

E. Subcutaneous fat index

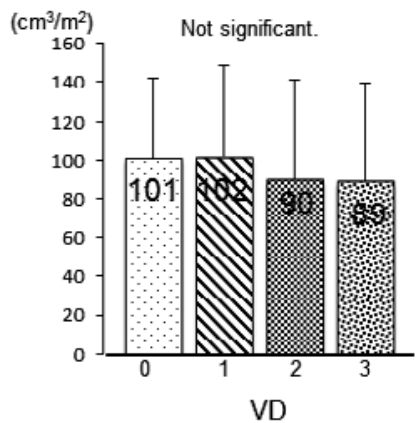

Figure 3. Pericardial fat (A), paracardial fat $(\mathbf{B})$, epicardial fat $(\mathbf{C})$, visceral fat $(\mathbf{D})$ and subcutaneous fat $(\mathbf{E})$ in the 0-VD, 1 -VD, 2-VD and 3-VD groups. $+P<0.05, * P<0.01, * * P<0.001, * * * P<0.0001$ versus 0 VD
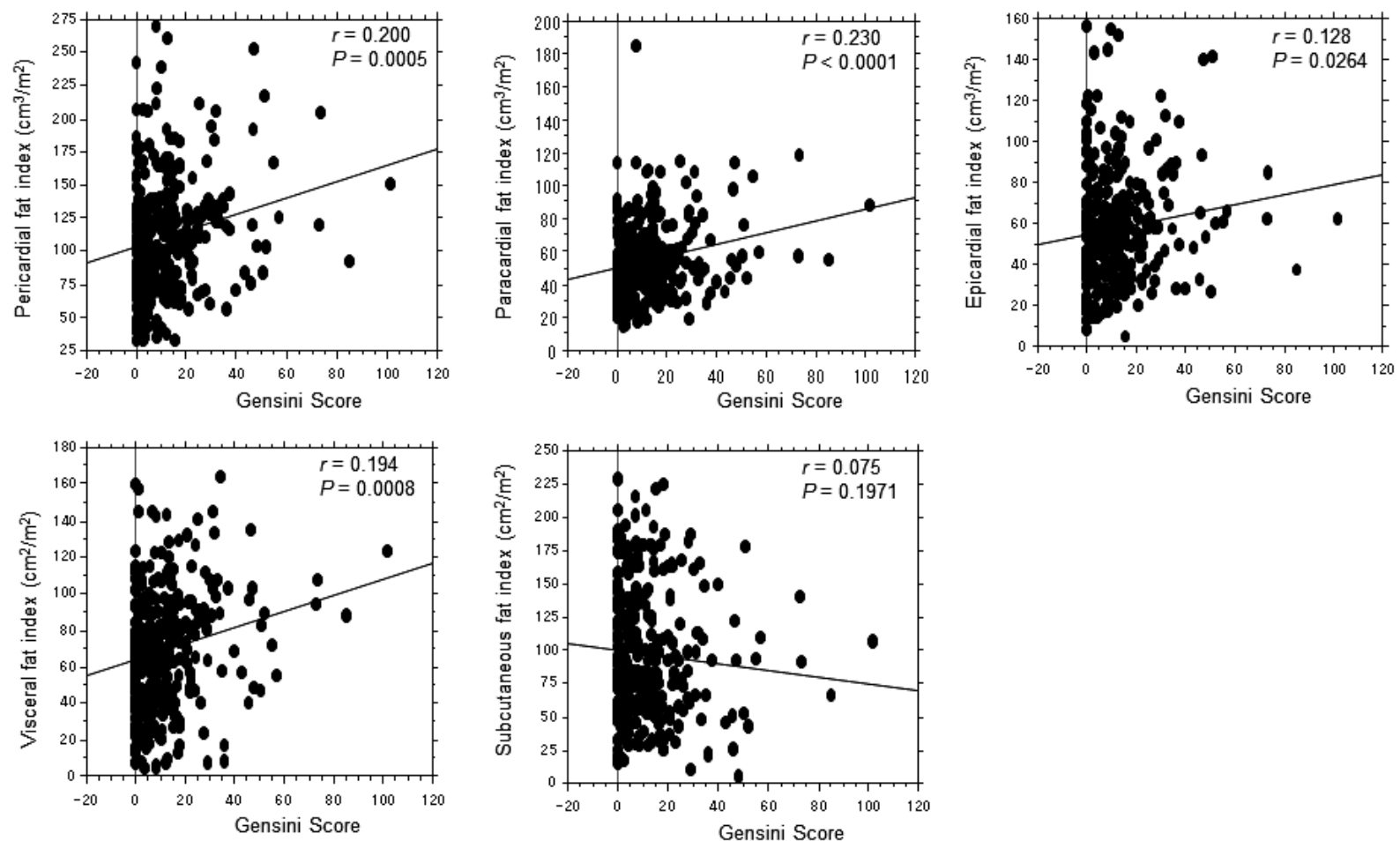

Figure 4. Association between pericardial fat, paracardial fat, epicardial fat, visceral fat or subcutaneous fat and the Gensini score. 
A.
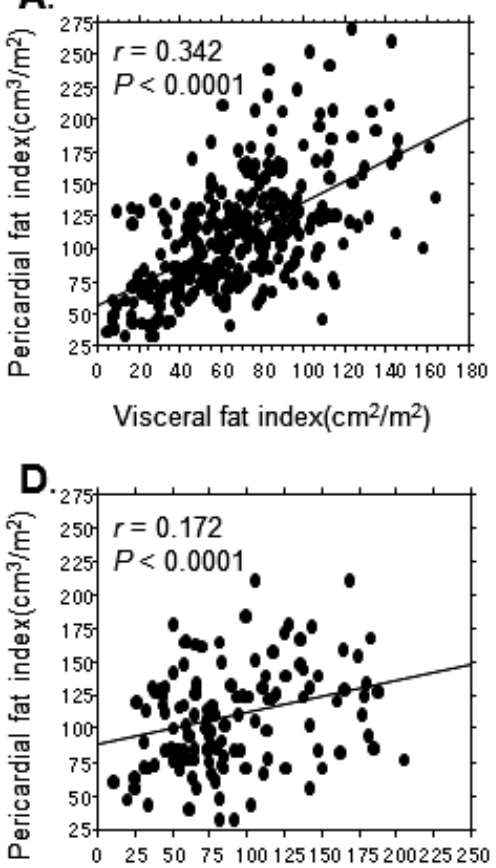

Subcutaneous fat index $\left(\mathrm{cm}^{2} / \mathrm{m}^{2}\right)$

G.

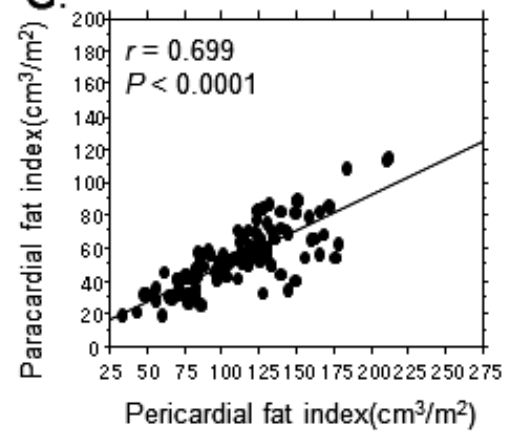

B.

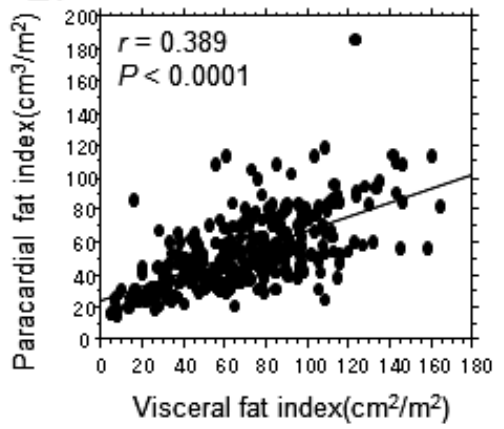

E.

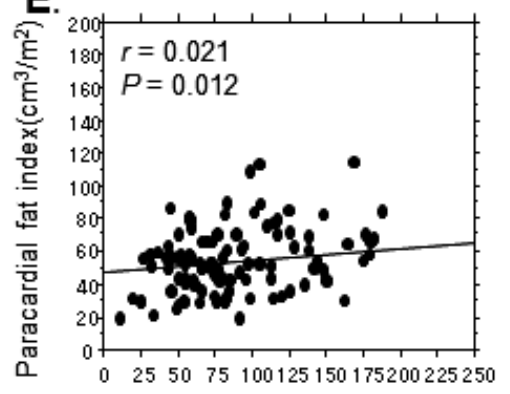

Subcutaneous fat index $\left(\mathrm{cm}^{2} / \mathrm{m}^{2}\right)$

$\mathrm{H}$.

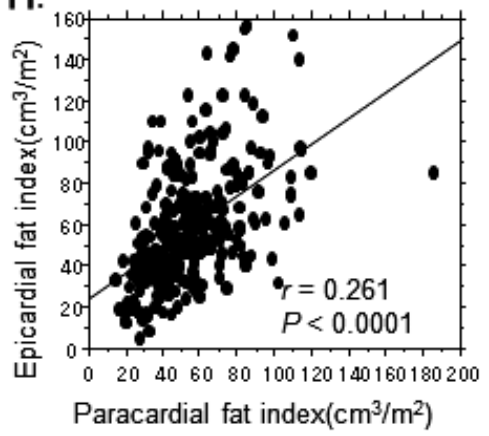

C.

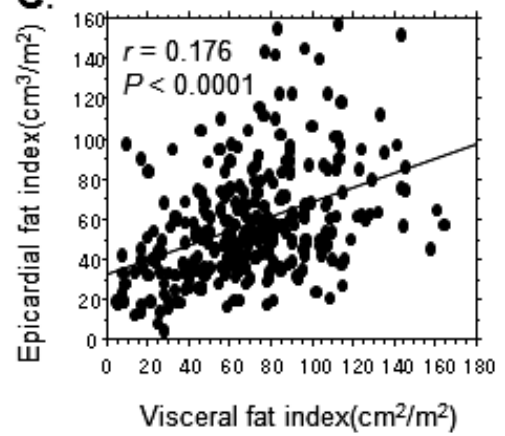

F.

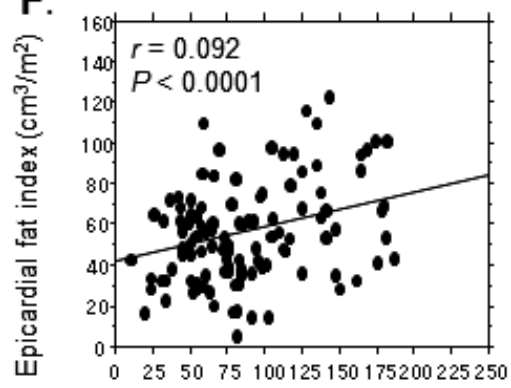

Subcutaneous fat index $\left(\mathrm{cm}^{2} / \mathrm{m}^{2}\right)$

I.

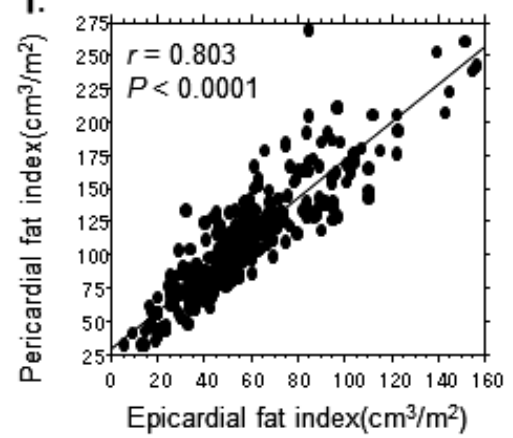

Figure 5. Association between pericardial fat, paracardial fat or epicardial fat and visceral fat or subcutaneous fat.

ness measured by echocardiography can be used as an independent marker to predict CAD. ${ }^{20)}$ They did not measure paracardial fat. Importantly, they analyzed the thickness, but not the volume, of pericardial fat. Furthermore, while they used echocardiography to measure fat values, we used CT. On the other hand, we analyzed 5 fat values (epicardial fat, pericardial fat, and paracardial fat in addition to visceral fat and subcutaneous fat) using CT. These other studies did not measure visceral fat or subcutaneous fat. Thus, there are many differences between our study and previous studies with regard to the methods used and the kinds of fat examined. Finally, we determined which of 5 kinds of fat was the most useful for predicting the presence of CAD. Thus, our report is the first to analyze the associations between 5 kinds of fat indexes and the presence or severity of coronary artery disease.

Epicardial fat is located between the epicardium and myocardium. Epicardial fat originates in the splanchnopleuric mesoderm and vascularization for epicardial fat is supplied by a branch of the coronary artery. ${ }^{21)}$ On the other hand, paracardial fat is located at the epicardium surface and originates in the primitive thoracic mesenchyme. ${ }^{21)}$ Vascularization for paracardial fat is supplied by non-coronary sources. In addition, visceral fat accumulates around abdominal viscera and inside intraabdominal solid organs. A previous study demonstrated an association between atherosclerosis and visceral fat, but not subcutaneous fat. ${ }^{22)}$ Visceral adipose tissue and adipose tissue resident macrophages produce more proinflammatory cytokines, such as tumor necrotic factor- $\alpha$ and interleukin- 6 , and less adiponectin. ${ }^{23,24}$ Epicardial fat looks like visceral fat that surrounds the heart and coronary arteries. Endocrine and paracrine activities, which lead to the secretion of pro-inflammatory and anti-inflammatory cytokines and chemokines, influence the development of coronary atherosclerosis. ${ }^{25-27)}$ Thus, epicardial fat is more important than paracardial fat for the progression of coronary atherosclerosis. Unexpectedly, paracardial fat was associated with 
A. Pericardial fat index

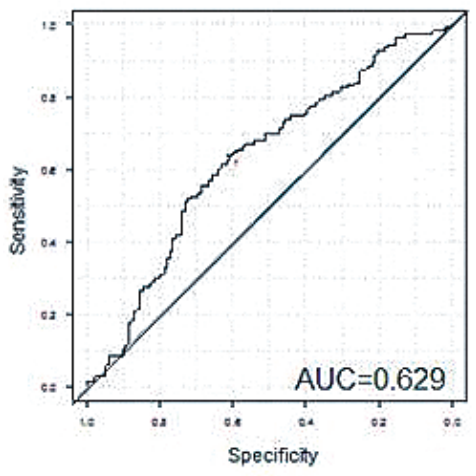

\section{Visceral fat index}

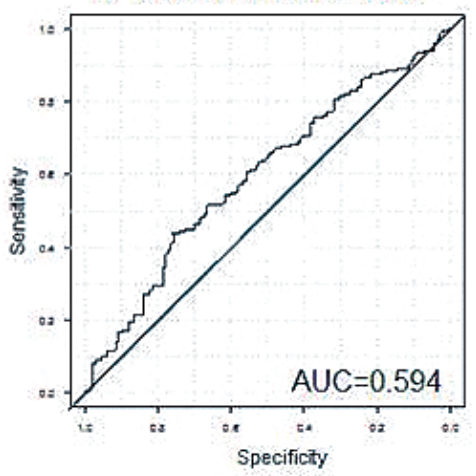

B. Paracardial fat index

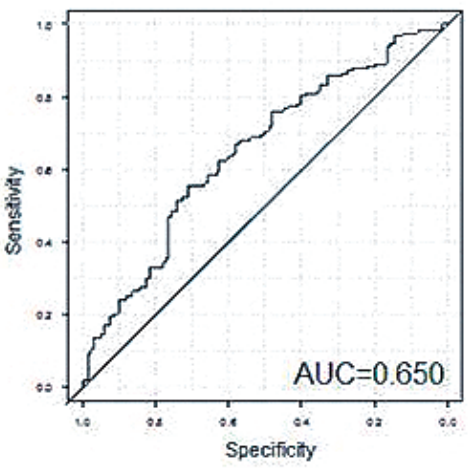

\section{E. Subcutaneous fat index}

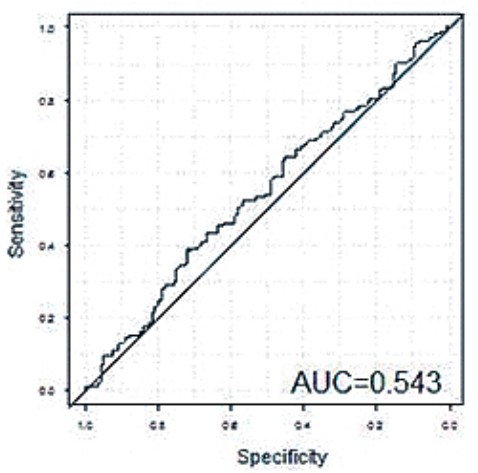

\section{Epicardial fat index}

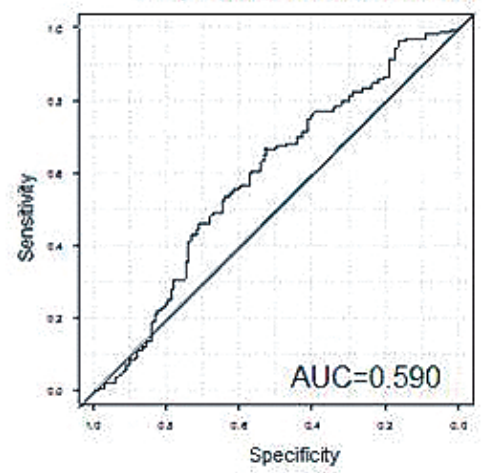

Figure 6. Cut-off values of pericardial fat, paracardial fat, epicardial fat, visceral fat and subcutaneous for the diagnosis of CAD.

Table III. Predictors for the Presence of CAD

\begin{tabular}{lcc}
\hline \multicolumn{1}{c}{ Factor } & OR $(95 \% \mathrm{CI})$ & $P$ \\
\hline Gender & $0.475(0.256-0.884)$ & 0.019 \\
Age & $1.057(1.028-1.086)$ & $<0.0001$ \\
BMI & $0.942(0.866-1.025)$ & 0.167 \\
HTN & $1.427(0.785-2.593)$ & 0.243 \\
DL & $1.275(0.732-2.222)$ & 0.391 \\
DM & $2.405(1.236-4.679)$ & 0.010 \\
Smoking & $1.186(0.839-1.677)$ & 0.333 \\
Paracardial fat index & $1.018(1.003-1.032)$ & 0.015 \\
\hline
\end{tabular}

CAD indicates coronary artery disease; OR, odds ratio; $\mathrm{CI}$, confidence interval; BMI, body mass index; HTN, hypertension; DL, dyslipidemia; and DM, diabetes mellitus.

the presence of CAD in this study. We do not know why there was a discrepancy between these results. Only one report showed an association between paracardial fat and coronary artery calcification in postmenopausal women, and this association was dependent on estradiol levels. The authors reported that were associations between lower levels and greater declines of estradiol and paracardial fat, but not epicardial fat. ${ }^{21)}$ Although it is not clear why estradiol levels modulated coronary artery calcification, paracardial fat may be a potential menopause marker for the risk of CAD. Further studies will be needed to clarify this issue.

Visceral fat has been reported to be associated with atherosclerosis. ${ }^{22)}$ In this study, while visceral fat levels in the CAD group were significantly higher than those in the non-CAD group (Figure 2), the presence of CAD was not independently associated with visceral fat index (Supplemental Table III). Since visceral fat index in the DM group $\left(79 \mathrm{~cm}^{2} / \mathrm{m}^{2}\right)$ was significantly higher than that in the non-DM group $\left(67 \mathrm{~cm}^{2} / \mathrm{m}^{2}\right)(P=0.005)$, the presence of CAD may not be associated with visceral fat index independent of DM.

Age and DM in addition to the paracardial fat level were independent predictors for the presence of CAD. Age and DM are well-known essential risk factors for CAD. This result indicated that the subjects in this study were not a specific patient group. The CAD group was significantly older and showed higher levels of \%male, prevalence of HTN, DM, DL, use of ARB/ACE-I, CCB, statin, $\beta$-blocker, SU, $\alpha$ GI and DPP-4I, visceral fat, MetS, hsCRP, pericardial fat, paracardial fat, and epicardial fat, and a significantly lower percentage of females and a lower level of HDL-C than the non-CAD group. It is reasonable that the CAD group had higher $\%$ medications for treatment than the non-CAD group because age, gender, HTN, DM, DL, and MetS are well-known essential risk factors for CAD. ${ }^{28-31)}$

Study limitations: This study has several important limitations. First, the sample size was relatively small, which limited our ability to determine significance. Second, MDCT is not a gold standard for the evaluation of CAD, although recent studies have shown that both its sensitivity and specificity were approximately $95 \%$ of those for 
invasive coronary angiography for the identification of significant coronary stenosis. ${ }^{26,32)}$ Third, although it was not very difficult to distinguish between paracardial and epicardial fat using 64-MDCT, we should obtain all fat data using the same MDCT. In addition, the need for an expert and the need for time to measure pericardial fat are drawbacks, and the development of analytical software is expected. A large-scale prospective study will be needed to address these issues.

\section{Conclusions}

Our results suggest that paracardial fat may be a marker for evaluating the presence or severity of CAD.

\section{Disclosures}

Conflicts of interest: K.S. is a Chief Director and S.M. is a Director of NPO Clinical and Applied Science, Fukuoka, Japan. K.S. has an Endowed Department of Molecular Cardiovascular Therapeutics, Fukuoka University, supported by MSD Co., Ltd. S.M. belongs to the Department of Molecular Cardiovascular Therapeutics supported by MSD, Co. LTD.

\section{References}

1. Examination committee of criteria for diagnosis of metabolic syndrome in Japan. Definition and criteria for diagnosis of metabolic syndrome. J Jpn Soc Int Med 2005; 94: 794-809. (Japanese)

2. Fox CS, Massaro JM, Hoffmann U, et al. Abdominal visceral and subcutaneous adipose tissue compartments: association with metabolic risk factors in the Framingham Heart Study. Circulation 2007; 116: 39-48.

3. Rosito GA, Massaro JM, Hoffmann U, et al. Pericardial fat, visceral abdominal fat, cardiovascular disease risk factors, and vascular calcification in a community-based sample: the Framingham Heart Study. Circulation 2008; 117: 605-13.

4. Kannel WB, Cupples LA, Ramaswami R, Stokes J 3rd, Kreger BE, Higgins M. Regional obesity and risk of cardiovascular disease; the Framingham Study. J Clin Epidemiol 1991; 44: 18390.

5. Rumberger JA, Sheedy PF 3rd, Breen JF, Schwartz RS. Coronary calcium, as determined by electron beam computed tomography, and coronary disease on arteriogram. Effect of patient's sex on diagnosis. Circulation 1995; 91: 1363-7.

6. Nitta K, Akiba T, Suzuki K, et al. Assessment of coronary artery calcification in hemodialysis patients using multi-detector spiral CT scan. Hypertens Res 2004; 27: 527-33.

7. Achenbach S, Ropers D, Hoffmann U, et al. Assessment of coronary remodeling in stenotic and nonstenotic coronary atherosclerotic lesions by multidetector spiral computed tomography. J Am Coll Cardiol 2004; 43: 842-7.

8. Taguchi R, Takasu J, Itani Y, et al. Pericardial fat accumulation in men as a risk factor for coronary artery disease. Atherosclerosis 2001; 157: 203-9.

9. Iacobellis G, Leonetti F. Epicardial adipose tissue and insulin resistance in obese subjects. J Clin Endocrinol Metab 2005; 90: 6300-2.

10. Mitsutake R, Niimura H, Miura S, et al. Clinical significance of the coronary calcification score by multidetector row computed tomography for the evaluation of coronary stenosis in Japanese patients. Circ J 2006; 70: 1122-7.

11. Gensini GG. A more meaningful scoring system for determining the severity of coronary heart disease. Am J Cardiol 1983; 51: 606.

12. Sayın MR, Çetiner MA, Karabağ T, Doğan SM, Aydın M, Yavuz N. The relationship between the Gensini score and complete blood count parameters in coronary artery disease. Kosuyolu Heart J 2012; 15: 51-4.

13. Mahabadi AA, Lehmann N, Kalsh H, Robens T, Bauer M, Dykun I, Budde T, Moebus S, Jockel K-H, Erbel R, Mohlenkamp S. Association of epicardial adipose tissue with progression of coronary artery calcification is more pronounced in the early phase of atherosclerosis. JACC Cardiovasc Imaging 2014; $7:$ :909-16.

14. The Japan Atherosclerosis Society. Chapter 3. Goals of Dyslipidemia Management. J Atheroscler Thromb 2009; 16 Supl: 1525.

15. American Diabetes Association. Screening for type 2 diabetes. Diabetes Care 2004; 27: S11-4.

16. de Vos AM, Prokop M, Roos CJ, et al. Peri-coronary epicardial adipose tissue is related to cardiovascular risk factors and coronary artery calcification in post-menopausal women. Eur Heart J 2008; 29: 777-83.

17. Bettencourt N, Toschke AM, Leite D, et al. Epicardial adipose tissue is an independent predictor of coronary atherosclerotic burden. Int J Cardiol 2012; 158: 26-32.

18. Alexopoulos N, McLean DS, Janik M, Arepalli CD, Stillman AE, Raggi P. Epicardial adipose tissue and coronary artery plaque characteristics. Atherosclerosis 2010; 210: 150-4.

19. Rodriguez-Granillo GA, Carrascosa P, Deviggiano A, et al. Pericardial fat volume is related to atherosclerotic plaque burden rather than to lesion severity. Eur Heart J Cardiovasc Imaging 2017; 18: 795-801.

20. Ghaderi F, Eshraghi A, Shamloo AS, Mousavi S. Association of epicardial and pericardial fat thickness with coronary artery disease. Electron Physician 2016; 8: 2982-9.

21. El Khoudary SR, Shields KJ, Janssen I, et al. Postmenopausal women with greater paracardial fat have more coronary artery calcification than premenopausal women: The Study of Women's Health Across the Nation (SWAN) Cardiovascular Fat Ancillary Study. J Am Heart Assoc 2017; 6: e004545.

22. Ohman MK, Wright AP, Wickenheiser KJ, Luo W, Eitzman DT. Visceral adipose tissue and atherosclerosis. Curr Vasc Pharmacol 2009; 7: 169-79.

23. Iacobellis G, Bianco AC. Epicardial adipose tissue: emerging physiological, pathophysiological and clinical features. Trends Endocrinol Metab 2011; 22: 450-7.

24. Hamdy O, Porramatikul S, AI-Ozairi E. Metabolic obesity: the paradox between visceral and subcutaneous fat. Curr Diabetes Rev 2006; 2: 367-73.

25. Mazurek T, Zhang L, Zalewski A, et al. Human epicardial adipose tissue is a source of inflammatory mediators. Circulation 2003; 108: 2460-6.

26. Hirata Y, Tabata M, Kurobe H, et al. Coronary atherosclerosis is associated with macrophage polarization in epicardial adipose tissue. J Am Coll Cardiol 2011; 58: 248-55.

27. Liang Y, Yang C, Zhou Q, et al. Serum Monokine induced by gamma interferon is associated with severity of coronary artery disease. Int Heart J 2017; 58: 24-9.

28. Grundy SM, Pasternak R, Greenland P, Smith S, Fuster V. Assessment of cardiovascular risk by use of multiple-risk-factor assessment equations. Circulation 1999; 100: 1481-92.

29. Najafi M, Sheikhvatan M. Gender differences in coronary artery disease: correlational study on dietary pattern and known cardiovascular risk factors. Int Cardiovasc Res J 2013; 7: 124-9.

30. Marroquin OC, Kip KE, Kelley DE, et al; Women's Ischemia Syndrome Evaluation Investigators. Metabolic syndrome modifies the cardiovascular risk associated with angiographic coronary artery disease in women: a report from the Women's Ischemia Syndrome Evaluation. Circulation 2004; 109: 714-21.

31. Naito R, Miyauchi K. Coronary artery disease and type 2 diabetes mellitus. Int Heart J 2017; 58: 475-80. 
32. Ropers D, Rixe J, Anders K, et al. Usefulness of multidetector row computed tomography with $64-\mathrm{x} 0.6-\mathrm{mm}$ collimation and 330-ms rotation for the noninvasive detection of significant coronary artery stenoses. Am J Cardiol 2006; 97: 343-8.

\section{Supplemental Files}

Supplemental Tables I, II, and III

Please see supplemental files;

https://doi.org/10.1536/ihj.17-234 